Dental practice in the UK in 2015/2016. Part 2: aspects of direct restorations, bleaching, endodontics and paediatric dentistry.

\title{
Wilson, NHF
}

http://hdl.handle.net/10026.1/13196

10.1038/sj.bdj.2019.50

British Dental Journal

Springer Nature [academic journals on nature.com]

All content in PEARL is protected by copyright law. Author manuscripts are made available in accordance with publisher policies. Please cite only the published version using the details provided on the item record or document. In the absence of an open licence (e.g. Creative Commons), permissions for further reuse of content should be sought from the publisher or author. 
Dental practice in the UK in 2015/2016 - Part 2:

Aspects of direct restorations, bleaching, endodontics and paediatric dentistry
N.H. F. Wilson ${ }^{1}$
F.J.T. Burke ${ }^{2}$
P.A. Brunton ${ }^{3}$
S. Creanor ${ }^{4}$
M.T. Hosey ${ }^{5}$
F. Mannocci ${ }^{6}$

1. King's College London Dental Institute, London, UK

2. Primary Dental Care Research Group, University of Birmingham School of Dentistry, Birmingham, UK

3. Sir John Walsh Research Institute, Faculty of Dentistry, University of Otago, Dunedin, New Zealand

4. Medical Statistics, Plymouth University Peninsula Schools of Medicine \& Dentistry, Plymouth, UK

5. Paediatric Dentistry, King's College London Dental Institute, London, UK

6. Endodontology,

King's College London Dental Institute, London, UK

Correspondence to: Professor Nairn H F Wilson

Email: nairn.wilson@btinternet.com 


\section{In brief}

This paper reports data which helps identify changes and trends in the provision of direct restorations, bleaching, endodontics and paediatric dentistry in general dental practice in the UK.

\section{Objective}

To follow up related studies reported in 2004/2005 and 2011/2012 by investigating aspects of direct restorations, bleaching, endodontics and paediatric dentistry in general dental practice, as part of a survey of arrangements and procedures in the clinical practice of dentistry.

\section{Methods}

The data presented were extracted from the data obtained from a piloted, 121-question questionnaire distributed at random to general dental practitioners in the UK attending postgraduate meetings in $2015 / 2016$, with a wide distribution of locations. Percentages reported are based upon the number of respondents who answered each question, given that not all respondents answered all 121 questions included in the questionnaire.

\section{Results}

Between 2008 and 2015, composite displaced amalgam as the material most commonly used by general dental practitioners (GDPs) for the restoration of two surface cavities in premolars and permanent molar teeth. Only $24 \%$ of respondents were of the view that dental amalgam should continue to be used freely, but not because of environmental or mercury toxicity concerns. In applying minimum intervention dentistry approaches, repair rather than replacement was considered by most GDPs for the management of defective restorations, irrespective of the material forming the restoration. The provision of homebased, vital bleaching had increased since 2008, with tooth sensitivity being the most commonly reported, unwanted, side effect. A small majority of respondents (54\%) were of the view that facial soft tissue aesthetics should be considered the practice of dentistry. Rubber dam was widely used in endodontic procedures (85\%) but not for other procedures. Rotary instrumentation was routinely used in root canal treatment by $88 \%$ of the respondents. A greater percentage of respondents indicated that they would use a preformed metal crown to restore primary molars either routinely or occasionally, compared to eight years ago (35\% cf. $23 \%$ ). Over three quarters of the respondents had heard of the Hall crown technique, and $50 \%$ of them had used it to good effect in their practices.

\section{Conclusion}

Key aspects of general dental practice in the UK changed between 2008 and 2015, highlighting the dynamic nature of clinical practice and the scope of practice of dentistry. Studies of the type reported are considered important in investigating trends and developments in dentistry.

\section{Key words}


General dental practice, Direct restorations, Bleaching (tooth whitening), Endodontics, Rubber dam, Paediatric dentistry

\section{Introduction}

This is the second of three papers reporting the findings of the third round of an investigation designed to provide the changing profile of aspects of general dental practice in the UK. The other two papers report demographic and practice arrangements ${ }^{1}$ and aspects of indirect restorations and prosthodontics ${ }^{2}$ respectively. The data presented in this paper were collected in 2015/2016, seven years after obtaining the data included in the papers reporting the second round of the investigation ${ }^{3-5}$, which took place in 2008, five years after the initial round in $2003^{6-8}$. The present paper reports on aspects of direct restorations, endodontics and bleaching in general dental practice.

\section{Methods}

As described in the first paper in the present series ${ }^{1}, 500$ hard copies of a piloted questionnaire, comprising 121 questions, were distributed in a random manner to dentists in general dental practice (GDPs) in the UK attending a wide variety of postgraduate courses across the UK, at which at least one of two of the authors (NHFW and FJTB) was presenting or otherwise contributing to in some way. This aimed to recruit study participants with a wide geographic distribution from across the UK, who expressed a willingness to complete and return the questionnaire, using stamped addressed envelopes provided, together with a covering letter, with the questionnaires. There were no inclusion or exclusion criteria for the study, other than the participating dentists confirming that they were in general dental practice in the UK. Many elements of the questionnaire were taken from the questionnaires used in the 2003 and 2008 surveys $^{3,6}$, but with additions to investigate aspects of the clinical practice of dentistry in the UK considered to have changed in the intervening years.

The data obtained from completed questionnaires were collated and analysed using Stata SE version 14. Percentages reported are based upon the number of respondents who answered each question, given that not all respondents answered all 121 questions included in the questionnaire.

\section{Results}

As detailed in the first paper in the current series ${ }^{1}$, a total of 388 useable questionnaires were returned from the 500 distributed - a response rate of $78 \%$.

Restorative materials - posterior teeth 
The restorative material used most commonly by the respondents for the restoration of occluso-proximal (Class II) cavities in premolar teeth was direct resin composite (composite; $66 \%, n=253$ ), with dental amalgam reported to be used by $40 \%$ of respondents $(n=155)$. The corresponding figures for occluso-proximal cavities in permanent molar teeth were composite $48 \%(n=184)$ and dental amalgam $55 \%(n=123)$. Cast gold, ceramic and composite inlays, let alone other materials such compomer type materials, were most commonly used in occluso-proximal situations by small numbers of respondents $(<3 \%, n<10$,$) in each case,$ especially in permanent molar teeth.

The selection of material to restore an occluso-proximal cavity was principally based on minimum intervention considerations $(59 \%, n=226)$, material durability $(53 \%, n=205)$, aesthetics and patient's preference $(48 \%, n=184$ and $46 \%, n=178$, respectively) and potential occlusal loading $(38 \%, n=146)$. Environmental concerns $(3 \%, n=12)$ and concerns about mercury toxicity by dentists $(1 \%, n=4)$ were the least important factors. Patient concerns about mercury toxicity were flagged up by $10 \%(n=39)$ of respondents. Time taken to complete to complete the restoration of the tooth was a concern to $11 \%$ of respondents $(n=43)$.

Further questioning on the continuing use of dental amalgam revealed that only $25 \%$ of respondents ( $n=95$ ) were of the view that amalgam should continue to be used freely. Forty one percent ( $n=152$ ) indicated that the use of dental amalgam should be phased down, or phased out, with $31 \%$ of respondents $(n=115)$ indicating that they remained undecided about the future use of dental amalgam.

Repair of restorations

Repair rather than replacement of a restoration with a marginal defect was considered by most of the respondents, irrespective of the material forming the restoration: amalgam $82 \%$ of respondents $(n=315)$; composite - $86 \%$ of respondents $(n=330)$; glass-ionomer, including resin-modified glass-ionomer cements $-63 \%$ of respondents $(n=235)$; ceramic $61 \%$ respondents $(n=227)$, and gold or metal-ceramic $-59 \%$ of respondents $(n=218)$.

\section{Bleaching}

With $90 \%$ of respondents $(n=349)$ having indicated that they provided home-based, vital bleaching, together with $28 \%$ of respondents $(n=107)$ having provided practice-based bleaching, it was confirmed that bleaching was widely practised, with the use of homebased bleaching having apparently increased since the time of the 2008 survey $190 \%$ cf. $81 \%)$. Concurrently, the application of practice-based bleaching was found to have decreased (28\% cf. 35\%).

With both home- and practice-based bleaching, the most common, unwanted side effect reported was 'tooth sensitivity' (89\% of respondents, $n=314)$. Interestingly, practice-based 
bleaching related tooth sensitivity was found to have increased since the time of the previous (2008) survey (89\% cf. $71 \%)$. Soft tissue inflammation was the second most common side effect, experienced by $24 \%$ of those providing home-based, vital bleaching $(n=86)$ and $30 \%$ of those who provided practice-based bleaching $(n=32)$. Transient, postbleaching mottling and increased prominence of mottling which existed prior to bleaching was an additional comment made by a small number of respondents $(n<10)$.

Facial soft tissue aesthetics

Respondents were divided on whether facial soft tissue aesthetics should be considered part of the practice of dentistry, with $54 \%$ of respondents $(n=206)$ indicating that it should cf. $46 \%,(n=176)$ who indicated that it should not.

\section{Endodontics}

Five percent of respondents $(n=18)$ indicated that they did not carry out molar endodontics, citing difficulty $(n=4)$, time required $(n=5)$, poor success rate $(n=1)$, inadequate remuneration $(n=3)$ and risk of complaints $(n=3)$ as reasons.

Sixty percent of respondents $(n=220)$ indicated that they typically complete root canal therapy of vital anterior teeth in one visit, with only $1 \%$ of respondents $(n=5)$ indicating that they typically took more than two visits for such therapy. The remaining $39 \%(n=140)$ respondents typically took two visits. In contrast, $27 \%(n=97)$ of respondents indicated that they completed root canal therapy of vital multi-rooted posterior teeth in one visit, with $12 \%$ of respondents $(n=45)$ indicating that they typically took more than two visits to complete such therapy. Fifty seven percent of respondents $(n=209)$ typically took two visits. The corresponding findings for non-vital teeth were: anterior teeth - typically one visit (36\%, $n=132)$, typically two visits $(61 \%, n=221)$ and more than two visits $(3 \%, n=11)$; multi-rooted posterior teeth - typically one visit $(17 \%, n=63)$, typically two visits $(62 \%, n=225)$ and more than two visits $(18 \%, \mathrm{n}=65)$.

Concerning the cleaning and shaping of root canals, $12 \%$ of respondents $(n=44)$ used manual filing, $37 \%(n=136)$ engine rotary techniques and $51 \%(n=187)$ a combination of rotary and manual instruments.

Obturation was achieved by means of cold lateral condensation of gutta percha (GP) by $68 \%$ of respondents $(n=255)$, warm lateral condensation of GP by $15 \%$ of respondents $(n=57)$, or using other techniques and materials $(12 \%, n=46)$.

Cone beam CT was used as an aid to endodontic diagnosis often by $<1 \%$ of respondents $(n=3)$ or sometimes by $7 \%$ of respondents $(n=27)$. Ninety two percent of respondents $(n=345)$ did not use cone beam CT in endodontic diagnosis. Where cone beam CT was used for endodontic diagnosis, it was considered to aid in the imaging of root resorption $(n=12)$, 
calcified canals $(n=6)$, aetiology of pain $(n=8)$ or, amongst other reasons, root anatomy, perforations and relationships to adjacent structures.

Rubber dam

Rubber dam was routinely used for endodontics $(85 \%, n=329)$, operative dentistry (31\%, $n=120)$, fissure sealing $(7 \%, n=28)$ and practice-based bleaching $(11 \%, n=42)$. Thirteen percent of respondents $(n=50)$ indicated that they did not use rubber dam.

Paediatric dentistry

Where an occluso-proximal restoration was indicated clinically in a primary molar tooth, the material most frequently selected was glass-ionomer cement (GIC; $55 \%, n=204$ ), followed by resin modified (RM) GIC (RMGIC; 32\%, $n=120)$, dental amalgam (9\%, $n=35$ ) and compomer $(54 \%, n=17)$. A few respondents $(n<10)$ indicated in additional comments that composite could be selected for this application.

As an alternative to the placement of a direct, compound restoration in deciduous molars, six percent of respondents $(n=21)$ routinely used a preformed metal crown (PMC) - an approach used occasionally by a further $29 \%$ of respondents $(n=109)$.

Seventy six percent of respondents $(n=285)$ indicated that they were aware of the Hall technique, and $50 \%(n=150)$ of these individuals had applied it clinically. Of these individuals, $83 \%$ ( $n=125$ ) had found the technique to be successful.

For the restorative management of hypoplastic defects in first permanent molar teeth, $67 \%$ of respondents ( $n=254)$ favoured the use of composite, GIC $(16 \%, n=63)$ or RMGIC $(18 \%$, $\mathrm{n}=67$ ), with compomer or dental amalgam being favoured by a minority of respondents both $4 \%(n=15)$.

\section{Discussion}

If nothing else, the findings presented in the present paper provide 'food for thought' for GDPs who find themselves at odds with the opinions and views expressed by the majority of fellow practitioners, assuming it is accepted that the sample of practitioners included in the present investigation is representative ${ }^{1}$. The findings are, however, considered to serve other purposes, ranging from helping to map out trends and the resultant consequences in general dental practice to help identify priorities for research, especially practice-based research of immediate, practical relevance. That said, it is acknowledged that findings from surveys of the type undertaken may suffer limitations, including some respondents, possibly with the best of intentions, giving answers reflecting what they think is expected or correct, rather than what they personally believe or do in their day-to-day practice. Efforts were made to minimise such biased responses, including the letter, which accompanied the 
survey questionnaire, stressing that all responses would be anonymous, with no opportunity to identify individual respondents. On a more positive note, the response rate of $77.6 \%$ is good, especially given the length of the questionnaire (121 questions), the time taken to complete it (15-20 minutes) and the absence of any personal incentive for respondents.

Restorative materials for posterior teeth

Between the time of the previous survey in 2008 and the collection of the data presented in the present paper in 2015, there would appear to have been a 'watershed' shift in the material most commonly used to restore an occluso-proximal cavity in both premolar and permanent molar teeth. In the 2008 survey, the majority of respondents indicated that the material they used most commonly in the restoration of two-surface, occluso-proximal cavities in premolars (59\%) and permanent molars (75\%) was dental amalgam. As reported above, the corresponding figures in 2015 were $40 \%$ and $55 \%$ respectively, with composite having replaced dental amalgam as the material used most commonly by most GDPs for the restoration of two-surface cavities in premolar teeth - an historic turning point in conservative (operative) dentistry in the UK, with the possible exception of Wales where a recent survey indicated that amalgam remains the material typically selected for the restoration of posterior teeth in adults receiving NHS care ${ }^{9}$. It was noted with interest that environmental issues and concerns by both GDPs and patients about mercury toxicity were not important factors in this substantial shift in the selection of material for the restoration of posterior teeth. Also, restoration placement time was not an issue for the clear majority of the respondents $(<89 \%)$, possibly putting an end to the view that placement time is a barrier to composite being selected as the material of choice for the restoration of posterior teeth - a beneficial effect of, amongst other innovations, improved matrix systems, adhesives, curing depths and finishing systems. Further reasons for this shift in thinking may include, in addition to increasing patient expectations for tooth-coloured restorations, the rapidly increasing percentage of the dental workforce which learnt to use composite before dental amalgam in the restoration of posterior teeth while at dental school ${ }^{10}$.

It was considered most encouraging that minimum intervention considerations were found to be the factor which influenced the greatest percentage of respondents (59\%) in their choice of material for the restoration of posterior teeth. The messages that it is better to fill minimum intervention cavities with composite rather than dental amalgam, which is difficult to condense in cavities of reduced size, with limited access and rounded internal architecture $^{11}$, and, secondly, that the clinical performance of relatively small posterior composites may be found to be at least as good, if not better than that of larger, more interventive restorations of dental amalgam, would appear to have been put into practice by many practitioners.

It was of note also that only $25 \%$ of the respondents were of the view that dental amalgam should continue to be used freely, with a further $30 \%$ of the respondents being undecided about the future use of dental amalgam. 
Given the above findings, it is suggested, as recommended by Lynch and Wilson ${ }^{12,13}$ that plans should be in place to support the relatively rapid reduction in the use of dental amalgam in the restoration of premolars and permanent molars in clinical practice, in addition to the recent discontinuation of the use of dental amalgam in children and pregnant and lactating mothers ${ }^{14}$.

Another hugely encouraging aspect of the present study was the finding that the repair rather than replacement of a restoration with a marginal defect was considered by the majority of the respondents, irrespective of the material forming the restoration. With so many practitioners indicating that they would consider repairing rather than replacing a restoration with a marginal defect, it must be concluded that, as taught in most dental school the adage 'if in doubt take it out' (right back to sound dentine) can probably now be assigned to history ${ }^{15}$. Repair wherever possible, with total replacement as a last resort, would appear to be the thinking which is now gaining substantial traction in decision making in general practice. In addition, patients may be found to be more accepting of repair rather than replacement for the management of restorations found to have some form of limited defect ${ }^{15}$.

\section{Bleaching}

With $90 \%$ of respondents having indicated that they provided home-based, vital bleaching, and $38 \%$ of respondents having provided practice-based bleaching, the findings confirm that bleaching is as an element of everyday practice. As such, bleaching ought to taught at all dental schools, especially given the prevalence of the unwanted side effect of tooth sensitivity which is difficult to manage ${ }^{16}$. Amongst the many, different questions which could be asked in future surveys of arrangements and procedures in general dental practice, it would helpful to request more information on bleaching; for example, the typical age of patients who receive bleaching, difficulties in managing bleaching related tooth sensitivity, and experience of any long term consequences of bleaching, including difficulties in meeting older patients expectations of bleaching, especially if they were successfully bleached at a younger age. In the meantime, it was noted with interest that the percentage of respondents who indicated that they used practice-based bleaching had decreased since the time of the 2008 survey ( $28 \%$ cf. $35 \%$ ), and that the percentage of respondents who used practice-based bleaching and experienced related tooth sensitivity had increased since the time of the previous survey ( $89 \%$ cf. $71 \%$ ). Given these findings, it is suggested that, for the foreseeable future, home-based, vital bleaching will be the 'mainstay' of tooth whitening in general dental practice in the UK. The findings indicate that transient, post-bleaching mottling and increased prominence of mottling which exists prior to bleaching is an uncommon side effect of bleaching in general dental practice in the UK. 
While the data obtained indicated that a small majority of respondents ( $54 \%$ vs $46 \%$ ) were of the view that facial soft tissue aesthetics should be the practice of dentistry, it is suggested that this view may now be more widely held. This raises issues as to the role of the dental regulator. Should the regulator regulate what dentists do, or make decisions as to what dentists are permitted to do? If a reasonable body of dentists consider facial soft tissue aesthetics to be the practice of dentistry, it is suggested that the regulator should then regulate it in the interests of protecting the public. The alternative approach whereby the regulator, now comprising substantial lay input, defines and, in the process, limits the scope of practice of dentistry, excluding, for example, facial soft tissue aesthetics, could be argued to be contrary to the best interests of patients.

\section{Endodontics}

By $2015 / 2016$, rotary instrumentation was found to have become integral to root canal treatment procedures provided by most (88\%) practitioners. As such, instruction in rotary instrumentation, including exposure to all instruments used in continuous rotation and reciprocation, should form part of contemporary teaching and clinical instruction in endodontics.

The number of root canal treatments completed in multiple visits remained high, suggesting that information on the risks associated with multi-visit root canal therapy, including reinfection of the root canal space and fracture of teeth left with intermediate medications and temporary restorations, should be emphasised in continuing professional development (CPD) programmes on endodontics.

\section{Rubber dam}

While the use of rubber dam would appear to have increased since the time of the previous survey - non-users having dropped from $29 \%$ to $13 \%$ - it is cause for concern that a significant minority of practitioners $(<15 \%)$ did not indicate using rubber dam for endodontics, let alone other procedures in which effective isolation may be critical to patient safety and clinical outcome. In the event of an endodontic instrument finding its way into the alimentary canal, trachea or a bronchus, there can be little defence if related radiographs, such as endodontic diagnostic length periapical images, do not include evidence of a rubber dam having been applied, typically a rubber dam clamp. The shift to the use of rotary instrumentation is not considered to have changed the indication for the use of rubber dam in endodontics, principally for the isolation it provides. It is acknowledged that rubber dam, for those who have never been able to master the technique, can be very frustrating and time-consuming. This, it is suggested, is no excuse for never using rubber dam. It is a technique which is well within the capabilities of all dental practitioners, and once mastered is typically found to offer many benefits, over and above patient safety, with a minimum of additional chairside time and cost. 
Paediatric dentistry

Regarding the findings pertaining to paediatric dentistry, GICs continue to be the material of choice for occluso-proximal restorations in primary molars - $55 \%$ of respondents reported that they would place a GIC restoration, almost identical to the previous studies. However, more dentists (35\%) indicated that they would use a PMC either routinely or occasionally, compared to eight years ago (23\%). These findings are considered to suggest that UK dentists favour techniques that do not require administering local anaesthesia to a child. In 2008 , the findings suggested that practitioners were keen to stabilise caries in children, and the data obtained from the 2015 survey supports this. Over three quarters of respondents had heard of the Hall crown technique, and $50 \%$ of them had used it in their practices to good effect. This uptake of the Hall crown stabilisation technique shows that UK dentists are updating their scope and knowledge on the management of caries in children. Perhaps an effect of contractual arrangements, or, in all probability, more newly qualified dentists disseminating and applying the knowledge and guidance they gained in dental school. Despite this, PMCs are still not the favoured restoration for the management of proximalocclusal caries, perhaps this is because PMCs are more expensive than GICs, and practices are still weighing up the benefit of the better aesthetics, but repeated replacement of GICs, against the poorer aesthetics, but better longevity of the $\mathrm{PMC}^{17}$.

With only $9 \%(n=35)$ respondents indicating that dental amalgam was the material they used most frequently for the restoration of a two-surface cavity in a deciduous molar, it is suggested that the recently introduced restrictions on the use of dental amalgam in children ${ }^{14}$ will have very little impact on everyday conservative (operative) dentistry in children with a deciduous dentition.

To restore hypoplastic first permanent molars in children, dentists favoured adhesive materials, especially composite, with very few choosing dental amalgam. Given that young dentists are used to adhesive techniques and materials, the results also begin to question how hypoplastic first permanent molars will be managed in future. Perhaps more dentists will opt to restore them, so that they will be maintained into adulthood, rather than extract them in childhood?

\section{Key findings}

Assuming the findings of the present study are considered representative of general dental practice at the time of the survey, the data obtained indicate the following:

1. Sometime between 2008 and 2105, composite overtook dental amalgam as the material most commonly used in the restoration of two-surface cavities in premolar teeth.

2. Only $24 \%$ of respondents were of the view that dental amalgam should continue to be used freely, but not because of environmental or mercury toxicity concerns. 
3. In applying minimum intervention dentistry approaches, repair rather than replacement was considered by the majority of the respondents for the management of defective restorations, irrespective of the material forming the restoration.

4. The use of home-based, vital bleaching has increased since 2008. Sensitivity was the most common unwanted side effect of tooth whitening.

5. Most respondents (54\%) were of the view that facial soft tissue aesthetics should be included in the practice of dentistry.

6. Rotary instrumentation and the risks of reinfection and fracture associated with multivisit endodontic therapy should be included and emphasised in the teaching and clinical instruction of endodontics.

7. Rubber dam should have been used more widely, specifically in endodontics.

8. More UK dentists know about and are using PMCs to stabilise carious primary molars, though GICs are still the most favoured material.

9.The recently introduced global restrictions on the use of dental amalgam will have very little impact on everyday conservative (operative) dentistry in children with a deciduous dentition, or hypoplastic first permanent molars.

\section{Conclusion}

Key aspects of general dental practice in the UK changed between 2008 and 2015, highlighting the dynamic nature of clinical practice and the scope of practice of dentistry. Studies of the type reported are considered important in investigating trends and developments in dentistry.

\section{Acknowledgement}

The authors wish to acknowledge the time given by all the respondents to complete the study's 121 question questionnaire.

\section{References}

1. Burke FJT, Wilson NHF, Brunton PA, Creanor S. Dental practice in the UK in 2015 Part 1: demographic and practice arrangements. Br Dent J 2017 (in submission).

2. Brunton PA, Burke, FJT, Wilson NHF, Creanor S, ?????. Dental practice in the UK in 2015 -Part 2: aspects of indirect restoration and prosthodontics. Br Dent J 2017 (in submission).

3. Brunton PA, Burke FJT, Sharif MO, Muirhead EK, Creanor S, Wilson NHF. Contemporary dental practice in the UK: demographic details and practicing arrangements in 2008. Br Dent J 2012; 212: 11-15.

4. Brunton PA, Burke FJT Sharif MO, Creanor S, Hosey MT, Mannocci F, Wilson NHF. Contemporary dental practice in the UK in 2008: aspects of direct restorations, endodontics and bleaching. Br Dent J 2011; 212:63-67. 
5. Brunton PA, Sharif MO, Creanor S, Burke FJT, Wilson NHF. Contemporary dental practice in the UK in 2008: indirect restorations and prosthodontics. Br Dent J 2012: 212: 115-129.

6. Burke FJT, Wilson NHF, Christensen GJ, Cheung SW, Brunton PA. Contemporary dental practice in the UK: demographic and practising arrangements. Br Dent J 2005; 198: 39-43.

7. Wilson NHF, Christensen GJ, Cheung SW, Burke FJT, Brunton PA. Contemporary dental practice in the UK: aspects of direct restorations, endodontics and bleaching. Br Dent J 2004; 197: 753-756.

8. Brunton PA, Christensen GJ, Cheung SW, Burke FJT, Wilson NHF. Contemporary dental practice in the UK: indirect restorations and fixed prosthodontics. Br Dent J 2005; 198: 99-130.

9. Lynch, CD, Farnell, DJJ, Stanton, H, Chestnutt, IG, Brunton, PA \& Wilson, NHF. No more amalgams: Use of amalgam and amalgam alternative materials in primary dental care. Br Dent J 2018; 225: 171-177.

10. Lynch, CD \& Wilson, NHF. The teaching of posterior resin composites: Planning for the future based on 25 years of research. J Dent 2014; 42: 503-516.

11. Duncalf, W.V. \& Wilson, N.H.F. Adaptation and condensation of amalgam restorations in Class II preparations of conventional and conservative design. Quintessence Int 1992; 23: 499-504.

12. Lynch CD, Wilson NHF. Managing the phase-down of amalgam. Part 1: Education and training issues. Br Dent J 2013; 215: 109-113.

13. Lynch CD, Wilson NHF. Managing the phase-down of amalgam. Part 2: Implications for practising arrangements and lessons from Norway. Br Dent J 2013; 215: 159-16

14. European Parliamentary Research Service. Mercury: aligning EU legislation with Minamata http://www.europarl.europa.eu/RegData/etudes/BRIE/2017/595887/EPRS_BRI(201 7)595887_EN.pdf

15. Wilson NHF, Lynch, CD, Brunton, PA, Hickel, R, Meyer-Lueckel, H, Gurgan, S, Pallesen, U, Shearer, AC, Tarle, Z, Cotti, E, Vanherle, G, Opdam. Criteria for the replacement of restorations: Academy of Operative Dentistry European Section. Op Dent 2016, Suppl. 7: S48-S57.

16. Greenwall L. Tooth sensitivity associated with tooth whitening. In: Tooth whitening techniques, Ed. L Greenwall. New York, CRC Press.

17. Innes NPT, Ricketts D, Chong L, Keightley AJ, Lamont T, Santamaria. Preformed crowns for managing decayed primary molar teeth in children.

Rhttp://www.cochrane.org/CD005512/ORAL_preformed-crowns-managing-decayedprimary-molar-teeth-children. 
